IPMU 09-0157

\title{
Primordial Black Holes as All Dark Matter
}

\author{
Paul H. Frampton ${ }^{(a, b)}$, Masahiro Kawasaki ${ }^{(a, c)}$, Fuminobu Takahashi ${ }^{(a)}$, \\ and Tsutomu T. Yanagida ${ }^{(a, d)}$ \\ (a) Institute for the Physics and Mathematics of the Universe, University of Tokyo, \\ Chiba 277-8582, Japan \\ (b) Department of Physics and Astronomy, University of North Carolina, Chapel Hill, \\ NC 27599-3255 \\ (c) Institute for Cosmic Ray Research, University of Tokyo, Chiba 277-8582, Japan \\ (d) Department of Physics, University of Tokyo, \\ Tokyo 113-0033, Japan
}

\begin{abstract}
We argue that a primordial black hole is a natural and unique candidate for all dark matter. We show that, in a smooth-hybrid new double inflation model, a right amount of the primordial black holes, with a sharply-defined mass, can be produced at the end of the smooth-hybrid regime, through preheating. We first consider masses $<10^{-7} M_{\odot}$ which are allowed by all the previous constraints. We next discuss much heavier mass $10^{5} M_{\odot}$ hinted at by entropy, and galactic size evolution, arguments. Effects on the running of the scalar spectral index are computed.
\end{abstract}




\section{Introduction}

The presence of dark matter (DM) has been firmly established by a host of observations, and its abundance was measured by the WMAP with an unprecedented precision:[1]

$$
\Omega_{\mathrm{DM}} h^{2}=0.1131 \pm 0.0034
$$

However it is not known yet what DM is made of, and the question remains a big mystery in modern cosmology as well as particle physics.

It is often claimed that there is no DM candidate in the framework of the standard model (SM), assuming that DM is made of elusive particles which have evaded all conventional DM searches. The DM particle must be electrically neutral, long-lived, and cold, but no such particle exists in the SM. Thus we need to postulate a theory beyond SM and introduce a new degree of freedom, which is usually made stable by imposing an additional discrete symmetry. The introduction of such a discrete symmetry may be motivated by other phenomenological reason. For instance, in the supersymmetric standard model (SSM), it is customary to introduce an R-parity in order to forbid dangerous operators which would give rise to too fast proton decay. Once the R-parity is imposed, the lightest supersymmetric particle (LSP) becomes stable, and therefore a DM candidate. On the other hand, there is an argument that the R-parity violation may be a common phenomenon in the string landscape [2]. If so, the dangerous operators must be absent due to some other reason(s) and the lifetime of LSP in the SSM may be too short to account for the DM.

If the DM is made of a weakly interacting massive particle (WIMP), we may be able to observe collider, direct and indirect DM signatures; the DM particles may be produced at LHC, and the next-generation direct search experiments will probe a significant portion of parameter space predicted by various theoretical DM models. In spite of thorough DM searches using widely different techniques, the results are negative so far 1 If no DM signature is found in the future experiments, it may suggest that the basic assumption that the DM is made of unknown particles is simply wrong.

\footnotetext{
${ }^{1}$ One of the exceptions is the DAMA experiment $[3$. However, it is still controversial concerning the interpretation of the experimental data. Very recently, two DM-like events were found by the CDMS II experiment 4, but more data is clearly necessary to draw definite conclusions.
} 
There actually is a DM candidate in the framework of SM, namely, a PBH [5]. In the early Universe PBHs can form when the density perturbation becomes large, and it has been known that a PBH of mass greater than $10^{15} \mathrm{~g}$ survives the Hawking evaporation [6] and therefore contributes to the DM density [7].

In consideration of the entropy of the universe it was pointed out in Ref. [8] that if all DM were in the form of $10^{5} M_{\odot}$ black holes it would contribute a thousand times more entropy than the supermassive black holes at galactic centers and hence be a statistically favored configuration. Here we consider primordial black holes (PBHs) with masses from $10^{5} M_{\odot}$ to $10^{-8} M_{\odot}$ and, subject to observational constraints, any of these masses can comprise all DM although the entropy argument favors the heaviest $10^{5} M_{\odot}$ mass.

There are several ways to realize large density fluctuations leading to PBH formation. One is phase transition involving violent processes like bubble collision [9, 10, 11] or the collapse of string loops [12, 13, 14, 15]. As we will see in the next section, however, both scenarios have difficulties. Another possibility is the production of PBHs from density fluctuations generated during inflation. Since the blue spectrum with a spectral index $n_{s}>1$ is disfavored by the WMAP data [1], a single inflation may not be able to produce large density fluctuations at small scales unless some dynamics is introduced during inflation. On the other hand, the density fluctuations can be easily enhanced at small scales in a double inflation model, as first discussed in Ref. [16] in the context of the $\mathrm{PBH}$ formation.

In this letter we discuss a double inflation model that consists of a smooth-hybrid inflation [17] and a new inflation [18]. The smooth-hybrid new double inflation was studied in Ref. [19] in the context of explaining the large running spectral index suggested by the WMAP 1st year data [20]. In this set-up PBHs with a narrow mass distribution are formed as a result of an explosive particle production between the two inflations [21]. We will show that the $\mathrm{PBH}$ mass can take a wide range of values from $10^{-8} M_{\odot}$ up to $10^{5} M_{\odot}$. Also, the resultant PBH mass has a correlation with running of spectral index, which was roughly estimated in a semi-analytical method in Ref. 22. Here we numerically calculated the correlation, which can be tested by future observations.

The rest of the letter is organized as follows. In Sec. 2 we briefly review the PBH formation and evaporation, and the cosmological constraints on the PBH abundance. In 
Sec. 3, we discuss a realistic double inflation model, namely the smooth-hybrid new inflation model, and show that PBHs with masses satisfying current observational constraints are produced. In Sec. 4 we discuss related issues and give conclusions.

\section{$2 \quad \mathrm{PBH}$ formation and observational constraints}

The black hole mass and the formation epoch are related to each other due to the causality. In the early Universe, the mass contained in the Hubble horizon sets an upper bound on the PBH mass formed at that time. Assuming that the whole mass in the horizon is absorbed into one black hole, we obtain

$$
\begin{aligned}
M_{\mathrm{BH}} & =\frac{4 \pi \sqrt{3} M_{P}^{3}}{\sqrt{\rho_{f}}} \simeq 0.05 M_{\odot}\left(\frac{g_{*}}{100}\right)^{-\frac{1}{2}}\left(\frac{T_{f}}{\mathrm{GeV}}\right)^{-2} \\
& \simeq 1.4 \times 10^{13} M_{\odot}\left(\frac{g_{*}}{100}\right)^{-\frac{1}{6}}\left(\frac{k_{f}}{\mathrm{Mpc}^{-1}}\right)^{-2}
\end{aligned}
$$

where $M_{\mathrm{BH}}$ is the black hole mass, $M_{P} \simeq 2.4 \times 10^{18} \mathrm{GeV}$ is the reduced Planck mass, $M_{\odot} \simeq 2 \times 10^{33} \mathrm{~g}$ is the solar mass, $g_{*}$ counts the light degrees of freedom in thermal equilibrium, $\rho_{f}, T_{f}$ and $k_{f}$ are the energy density, the plasma temperature and the comoving wavenumber corresponding to the Hubble horizon at the formation, respectively. The radiation domination was assumed in the second equality.

As is well known, Hawking made a striking prediction about the evaporation of black holes; any black holes have a temperature inversely proportional to its mass and evaporates in a finite time $\tau_{\mathrm{BH}}[6]$,

$$
\tau_{\mathrm{BH}} \simeq 10^{64}\left(\frac{M_{\mathrm{BH}}}{M_{\odot}}\right)^{3} \mathrm{yr} .
$$

Thus the black holes with mass less than $10^{15} \mathrm{~g}$ must have evaporated by now. PBHs which remain as (a part of) DM must therefore be created at a temperature below $10^{9}$ $\mathrm{GeV}$. In the following we assume that PBHs account for all DM in our Universe.

The cosmological effects of PBHs have been extensively studied so far. While PBHs with masses below $10^{15} \mathrm{~g}$ are significantly constrained, it is very difficult to detect PBHs heavier than $10^{15} \mathrm{~g}$ because of negligible amount of the Hawking radiation. The MACHO [23] and EROS [24] collaborations monitored millions of stars in the Magellanic 
Clouds to search for microlensing events caused by MAssive Compact Objects (MACHOs) passing near the line of sight. The MACHO collaboration [25] excluded the objects in the mass range $0.3 M_{\odot}$ to $30 M_{\odot}$, and the latest result of the EROS-1 and EROS-2 [26] excluded the mass range $0.6 \times 10^{-7} M_{\odot}<M<15 M_{\odot}$, as the bulk component of the galactic DM. On the other hand, if we assume that the PBH formation occurs before the big bang nucleosynthesis (BBN) epoch, the PBH mass should be lighter than $10^{5} M_{\odot}$ (see Eq. (2) ). Therefore we consider PBHs with masses (i) $M_{\mathrm{BH}}<10^{-7} M_{\odot}$ and (ii) $30 M_{\odot}<M_{\mathrm{BH}}<10^{5} M_{\odot}$.

Let us comment on other existing constraints. The PBHs with masses heavier than $43 M_{\odot}$ were claimed to be excluded by the presence of wide binaries [27], but the question on the validity of the data used to set the limit was raised by Ref. 28]. Taking account of low averaged DM density experienced by the four binaries used in their analysis, the strong constraints set by the wide binaries were undermined. Recently, Ricotti, Ostriker and Mack investigated the effect of non-evaporating PBHs on the cosmic microwave background (CMB) spectrum and anisotropy and found that the PBHs with mass greater than $\sim 0.1 M_{\odot}$ cannot account for the bulk component of DM [29]. However, the authors made assumptions about accretion efficiency in obtaining strong limits on PBH abundances; if these assumptions are weakened, all DM could be PBHs for the masses we consider 2 .

The above observational constraints provide us with information on the PBH formation. If PBHs are produced at different times, the mass function tends to be broad, thereby making it difficult to be consistent with observations. In order to realize the $\mathrm{PBH}$ mass function with a sharp peak, most of the PBHs should be produced at the same time. Thus the production mechanism must involve such a dynamics that only the density fluctuation of a certain wavelength rapidly grows.

What kind of dynamics can create PBHs? First of all, density perturbation must become large for PBHs to be formed. There are several ways to realize large density fluctuations leading to the PBH formation. One is the phase transition which leads to violent processes like bubble collision [9, 10, 11] or the collapse of string loops [12, 13, 14,

\footnotetext{
${ }^{2}$ As pointed out in Ref. [29], the Bondi solution becomes invalid for the PBH of mass $M_{\mathrm{BH}} \gtrsim 10^{4} M_{\odot}$. In particular, the duty cycle is not well understood because of the complicated feedback effect. If the duty cyle is very small, the constraint of [29] can be weakened.
} 
15. However, in the case of the bubble collision, the bubble formation rate must be tuned to produce the $\mathrm{PBH}$, and the $\mathrm{PBH}$ produced from the strong loops tends to have a broad mass function. Another possibility is the production of PBHs from density fluctuations generated during inflation. In the standard picture of inflation, the inflation driven by a slow-rolling scalar field lasts for more than about 60 e-foldings to solve theoretical problems of the big bang cosmology. Then no dynamics for producing a sharp peak in the density perturbation is expected [30]. However, there is no a priori reason to believe that our Universe experienced only one inflationary expansion. Indeed, the cosmological gravitino or modulus problem can be relaxed if the energy scale of the last inflation is rather low, and it is then quite likely that there was another inflation before the last one. If the multiple inflation is a common phenomenon, we expect that explosive particle production between the successive inflation periods may produce a sharp peak in the density perturbation at the desired scales, which leads to the $\mathrm{PBH}$ formation at a later time. In the next section, we show that this is actually feasible using a concrete double inflation model.

\section{PBHs from preheating}

In this section we provide a double inflation model producing PBHs with a sharp mass function as an existing proof. The double inflation model [19, 21, 22] we adopt consists of two stages of inflation; the first inflation is realized by smooth hybrid inflation and the second one by new inflation. As shown below, the cosmologically relevant density fluctuations are generated during smooth hybrid inflation. After the first inflation, the inflaton and waterfall fields of the smooth hybrid inflation start to oscillate and decay into their quanta via self-coupling and mutual coupling of the two fields. The interesting point is that the decays of the scalar fields are largely enhanced through parametric resonance and hence the fluctuations of the scalar fields exponentially grow. This process is called preheating. During the preheating phase, only the fluctuations at a specific wavenumber corresponding to the inflaton mass rapidly grow, and those fluctuations finally turn into density fluctuations leading to the production of PBHs with a sharp mass function. The role of the second inflation is to stretch the density fluctuations generated during the 
first inflation and the subsequent preheating phase to cosmologically large scales. In the following, we briefly describe the smooth-hybrid new inflation model [for details, see Refs.[19, 21]].

The first inflation is realized by smooth hybrid inflation [31]. In Refs. [19, 21], the smooth hybrid inflation model is built in framework of supergravity and the superpotential and Kähler potential are given by

$$
\begin{aligned}
& W_{H}=S\left(\mu^{2}+\frac{(\bar{\Psi} \Psi)^{m}}{M^{2(m-1)}}\right) \quad(m=2,3, \ldots), \\
& K_{H}=|S|^{2}+|\Psi|^{2}+|\bar{\Psi}|^{2},
\end{aligned}
$$

where $S$ is the inflaton superfield, $\Psi$ and $\bar{\Psi}$ are waterfall superfields, $\mu$ is the inflation scale and $M$ is the cut-off scale which controls the nonrenormalizable term. From the above superpotential and Kähler potential together with phase redefinition and the Dflat condition, we obtain the scalar potential as

$$
V_{H}(\sigma, \psi) \simeq\left(1+\frac{\sigma^{4}}{8}+\frac{\psi^{2}}{2}\right)\left(-\mu^{2}+\frac{\psi^{4}}{4 M^{2}}\right)^{2}+\frac{\sigma^{2} \psi^{6}}{16 M^{4}},
$$

where $\sigma \equiv \sqrt{2} \operatorname{Re} S$ and $\psi \equiv 2 R e \Psi=2 R e \bar{\Psi}$. Here and in what follows we use the Planck unit $M_{P}=1$ and take $m=2$ for simplicity. Although the scalar potential (6) is derived in the framework of supergravity, one may start with (6) without assuming supersymmetry. The potential (6) has a true vacuum at $\sigma=0$ and $\psi=2 \sqrt{\mu M}$. For $\sigma \gtrsim \sqrt{\mu M} / 2$, however, the potential for $\psi$ has a $\sigma$-dependent minimum at

$$
\psi_{\min } \simeq \frac{2}{\sqrt{3}} \frac{\mu M}{\sigma}
$$

Note that $\psi$ quickly settles down at the minimum during inflation since its mass is larger than the Hubble parameter. Then we can integrated out $\psi$ and obtain the effective potential for $\sigma$ as

$$
V(\sigma)=\mu^{4}\left(1+\frac{\sigma^{4}}{8}-\frac{2}{27} \frac{\mu^{2} M^{2}}{\sigma^{4}}\right)=\mu^{4}+\frac{\mu^{4}}{8}\left(\sigma^{4}-\sigma_{d}^{4}\left(\frac{\sigma_{d}}{\sigma}\right)^{4}\right)
$$

where $\sigma_{d} \equiv \sqrt{2} / 3^{3 / 8}(\mu M)^{1 / 4}$. If the scalar potential is dominated by the first term, the inflaton $\sigma$ slow rolls and therefore inflation occurs. 
According to the WMAP 5yr data [1], the curvature perturbation $\mathcal{R}$, the spectral index $n_{s}$ and its running $d n_{s} / d \ln k$ at the pivot scale $k_{*}=0.002 \mathrm{Mpc}^{-1}$ are

$$
\begin{aligned}
\mathcal{R} & =4.9 \times 10^{-5}, \\
n_{s} & =1.031 \pm 0.055 \\
\frac{d n_{s}}{d \ln k} & =-0.037 \pm 0.028 .
\end{aligned}
$$

From the effective potential (8) we obtain

$$
\begin{aligned}
\mathcal{R} & =\frac{V^{3 / 2}}{\sqrt{3} \pi V^{\prime}}=\frac{\mu^{2}}{\sqrt{3} \pi}\left[\sigma_{*}^{3}+\sigma_{d}^{3}\left(\frac{\sigma_{d}}{\sigma_{*}}\right)^{5}\right]^{-1}, \\
n_{s}-1 & \simeq 2 \frac{V^{\prime \prime}}{V}=\left[3 \sigma_{*}^{2}-5 \sigma_{d}^{2}\left(\frac{\sigma_{d}}{\sigma_{*}}\right)^{6}\right], \\
\frac{d n_{s}}{d \ln k} & \simeq-2 \frac{V^{\prime \prime \prime} V^{\prime}}{V^{2}}=-3\left[\sigma_{*}^{3}+\sigma_{d}^{3}\left(\frac{\sigma_{d}}{\sigma_{*}}\right)^{5}\right]\left[\sigma_{*}+5 \sigma_{d}\left(\frac{\sigma_{d}}{\sigma_{*}}\right)^{7}\right],
\end{aligned}
$$

where $\sigma_{*}$ is the field value of the inflaton when the fluctuation corresponding to the pivot scale exits the Hubble horizon.

The fluctuation corresponding to the pivot scale $k_{*}$ exits the horizon at $t=t_{*}$ when $k_{*} / a\left(t_{*}\right)=H_{H}=\mu^{2} / \sqrt{3}\left(H_{H}\right.$ : hubble during the smooth hybrid inflation). Thus the scale factor $a_{*}=a\left(t_{*}\right)$ is given by

$$
\ln a_{*}=-2 \ln \mu-136 .
$$

The e-folding number between the horizon exit of the pivot scale and the end of the smooth hybrid inflation is estimated as

$$
N_{*}(\sigma)=\int_{\sigma_{e}}^{\sigma_{*}} d \sigma \frac{V}{V^{\prime}} \simeq\left\{\begin{array}{ll}
\frac{4}{3 \sigma_{d}^{2}}-\frac{1}{\sigma_{*}^{2}} & \left(\sigma_{*}>\sigma_{d}\right) \\
\frac{\sigma_{*}^{6}}{3 \sigma_{d}^{8}} & \left(\sigma_{*}<\sigma_{d}\right)
\end{array},\right.
$$

where $\sigma_{e}\left(\ll \sigma_{d}\right)$ denotes the field value when the smooth hybrid inflation ends.

After the smooth hybrid inflation, $\sigma$ and $\psi$ oscillate about their minima and decay into the $\sigma$ and $\psi$ quanta via self-couplings and mutual coupling of the two fields. Since their effective masses depend on the field amplitudes and therefore time-dependent, specific modes of the $\sigma$ and $\psi$ quanta are strongly amplified by parametric resonance. To see this, 
let us write down the evolution equation for the Fourier modes of fluctuations $\sigma_{k}$ from (6) as

$$
\sigma_{k}^{\prime \prime}+3 H \sigma_{k}^{\prime}+\left[\frac{k^{2}}{a^{2}}+m_{\sigma}^{2}+3 m_{\sigma}^{2} \frac{\tilde{\psi}}{\sqrt{\mu M}} \cos \left(m_{\sigma} t\right)\right] \sigma_{k} \simeq 0,
$$

where $m_{\sigma}=\sqrt{8 \mu^{3} / M}$ and $\tilde{\psi}$ is the amplitude of the $\psi$ oscillations. $(\tilde{\psi} \sim \sqrt{\mu M}$ at the beginning of the oscillations.) Neglecting the cosmic expansion, Eq. (17) has a form similar to the Mathieu equation which is known to have a exponentially growing solution. The detailed numerical simulation showed that the wave number for the fastest growing mode is given by [21]

$$
\frac{k_{p}}{a_{\mathrm{osc}}} \simeq 0.3 m_{\sigma}
$$

The width of the peak is also determined by the instability band of Eq. (17), and it is of $O(0.1) m_{\sigma}$.

The fluctuations amplified by the parametric resonance eventually produce PBHs when they reenter the horizon after inflation. The mass of the $\mathrm{PBH}$ is approximately given by the horizon mass when the fluctuations reenter the horizon. Thus the PBH mass is estimated as

$$
M_{\mathrm{BH}} \simeq 1.4 \times 10^{13} M_{\odot}\left(\frac{k_{p}}{\mathrm{Mpc}^{-1}}\right)^{-2} .
$$

From Eqs. (18) and (19) the scale factor at the beginning of the oscillation phase is estimated as

$$
\ln a_{\mathrm{osc}}=-114-\ln m_{\sigma}-0.5 \ln \left(M_{\mathrm{BH}} / M_{\odot}\right) .
$$

Because the e-folding number $N_{*}$ is equal to $\ln a_{\text {osc }}-\ln a_{*}$, we obtain

$$
N_{*}=21+0.5 \ln (\mu M)-0.5 \ln \left(M_{\mathrm{BH}} / M_{\odot}\right)
$$

For a fixed black hole mass $M_{\mathrm{BH}}$, there are two parameters in the model, i.e., $\mu$ and $M$, one of which can be removed by using the WMAP normalization (9). Therefore observable quantities can be expressed in terms of one free parameter, leading to a non-trivial relation between $n_{s}$ and $d n_{s} / d \ln k$. In practice, we adopt $\mu M$ as the free parameter, and solve Eqs. (16) and (21) for $\sigma_{*}$ in terms of $\mu M$. Then $\mu$ and $M$ are determined with use of Eqs. (12) and (9) for a fixed $\mu M$. Thus, varying $\mu M$, we obtain sets of model parameters which are consistent with the observed curvature perturbations. 
After $\sigma$ and $\psi$ decay, the second inflation (= new inflation) starts. As mentioned before, the role of the new inflation is to stretch the fluctuations produced during the smooth hybrid inflation and subsequent preheating phase to appropriate cosmological scales. The effective potential for the new inflation is given by

$$
V_{\text {new }}=v^{4}\left(1-\frac{c}{2} \phi^{2}\right)-\frac{g}{2} v^{2} \phi^{4}+\frac{g^{2}}{16} \phi^{8}
$$

where $\phi$ is the inflaton of the new inflation, $v$ is the scale of the new inflation and $g$ and $c$ are constants. The scale factor $a_{f}$ at the end of the new inflation is estimated as

$$
\ln a_{f}=-68+\frac{1}{3} \ln \left(\frac{T_{R}}{10^{9} \mathrm{GeV}}\right)-\frac{4}{3}\left(\frac{v}{10^{15} \mathrm{GeV}}\right)
$$

where $T_{R}$ is the reheating temperature after the new inflation. Therefore, the new inflation should provide the total e-fold number $\simeq\left(\ln a_{f}-\ln a_{\mathrm{osc}}\right)$.

In order to estimate precisely the fluctuations generated in the present model, we numerically integrate the evolution equations for the homogeneous modes of $\sigma, \psi$, and $\phi$ and the Fourier modes of their fluctuations as well as the metric perturbations in the same method described in Ref. [21]. In Fig. 1 the power spectrum of the curvature perturbation $P_{\mathcal{R}}$ is shown for $\mu=4 v=7.14 \times 10^{-4}, M=0.29, c=0.1$ and $g=2 \times 10^{-5}$. It is seen that the spectrum has a very sharp peak at $k \simeq 10^{10} \mathrm{Mpc}^{-1}$ which corresponds to the PBH mass $\sim 10^{-7} M_{\odot}$. Since the peak is so sharp, PBHs with very narrow mass range are formed. The PBH abundance is determined by the height of the peak which depends on the decay rate of the inflaton. We can obtain an appropriate $\mathrm{PBH}$ abundance by tuning the decay rate [21]. We also predict the spectral index and its running for $M_{\mathrm{BH}}=10^{5} M_{\odot}, 10^{-7} M_{\odot}$ and $10^{-8} M_{\odot}$ in Fig. 2. Note that the e-folding number of the smooth hybrid inflation is smaller than that needed to solve the horizon and flatness problems by a single inflation model. Therefore the observable density fluctuations are produced near the end of the inflation when the slow roll parameters are larger, leading to a larger value of $d n_{s} / d \ln k$.

\section{Discussion and Conclusions}

In contrast to the conventional WIMP DM model, PBHs have only gravitational interactions. In order to detect PBHs, we need to carefully look at the effect induced by PBHs 


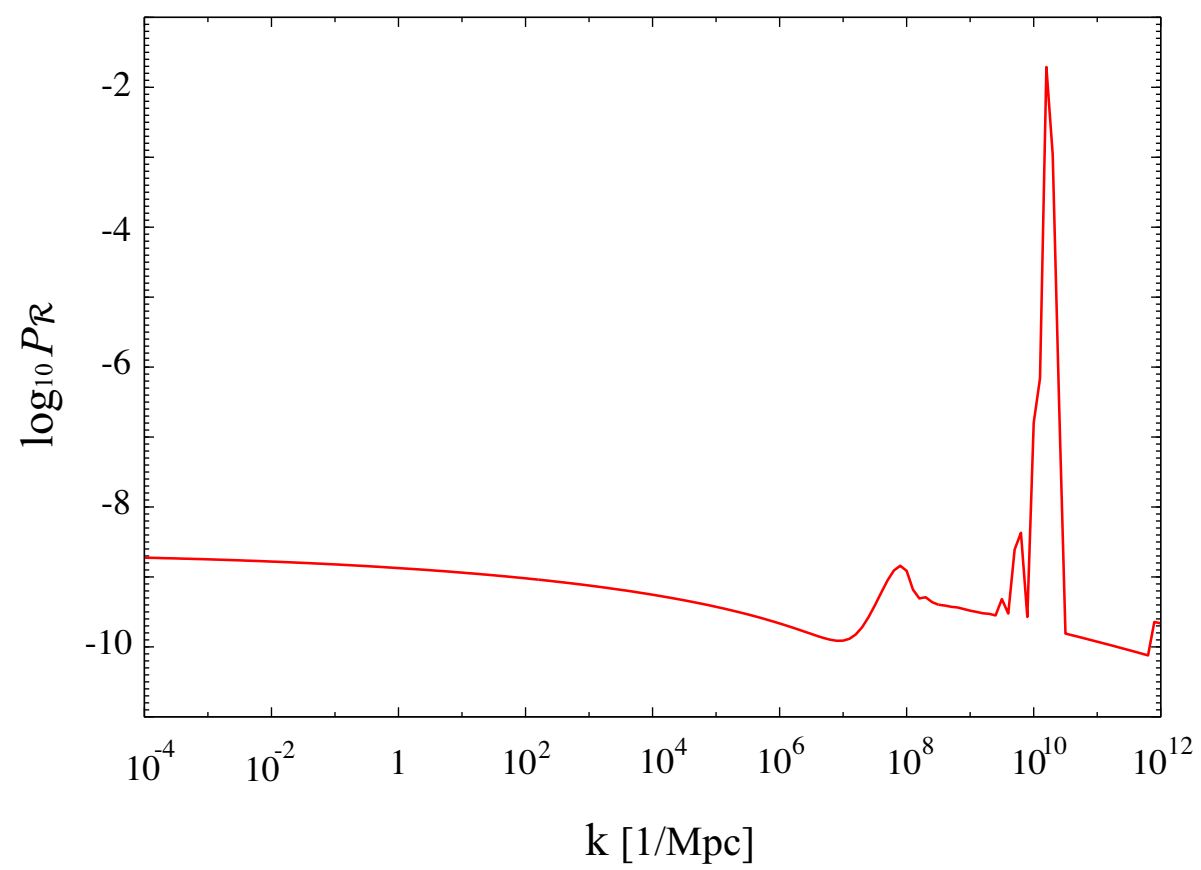

Figure 1: Power spectrum of the curvature perturbation. We take $\mu=4 v=7.14 \times 10^{-4}$, $M=0.29, c=0.1$ and $g=2 \times 10^{-5}$.

such as gravitational lensing, gravity waves, etc. Intermediate mass black holes in the range $30 M_{\odot}<M_{B H}<10^{5} M_{\odot}$ can be sought, for example, by higher-longevity microlensing events [23, 24] and by higher-statistics analysis of wide binaries [27, 28]. In particular there appeared recently an interesting idea that if the DM is explained by the $\mathrm{PBH}$ of mass $10^{5} M_{\odot}$, it may account for the size evolution of the elliptic galaxies by dynamical friction [32]. Further observations and theoretical study may reveal the presence of the PBH DM.

What makes the $\mathrm{PBH}$ particularly attractive as a DM candidate is that it is naturally long-lived due to the gravitationally suppressed evaporation rate. No discrete symmetries need to be introduced in an ad hoc manner. Also the PBH DM may be motivated from the arguments based on entropy of the Universe [8].

In this letter we have argued that the $\mathrm{PBH}$ is a natural and unique candidate for the DM in the minimal theoretical framework, namely, the SM. Using the smooth-hybrid new double inflation model, we have shown that it is possible to produce PBHs of mass 


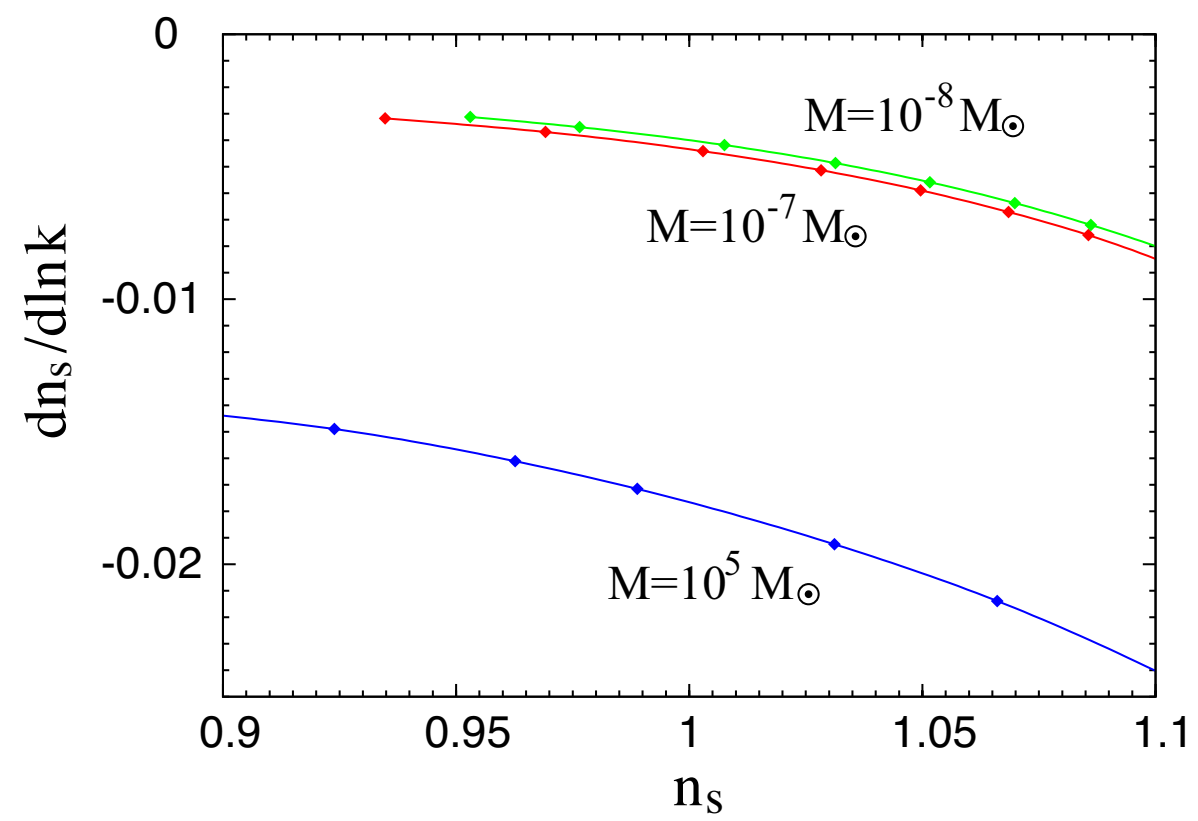

Figure 2: $n_{s}$ and $d n_{s} / d \ln k$ for PBH mass $M_{\mathrm{BH}}=10^{5} M_{\odot}, 10^{-7} M_{\odot}$, and $10^{-8} M_{\odot}$.

ranging from $10^{-8} M_{\odot}$ to $10^{5} M_{\odot}$. Importantly, the PBH mass relates the scalar spectral index and the running of the spectral index, which can be tested by the Planck satellite.

\section{Acknowledgment}

MK thanks Tsutomu Takayama for kindly providing his numerical code. The work of PHF was also supported in part by the U.S.Department of Energy under Grant No. DE-FG0206ER41418. The work of FT was supported by the Grant-in-Aid for Scientific Research on Innovative Areas (No. 21111006) and JSPS Grant-in-Aid for Young Scientists (B) (No. 21740160). This work was supported by World Premier International Center Initiative (WPI Program), MEXT, Japan.

\section{References}

[1] J. Dunkley, et al., Astrophys. J. Suppl. 180, 306 (2009) arXiv:0803.0586v1 [astro-ph]]. 
[2] R. Tatar and T. Watari, Nucl. Phys. B 747, 212 (2006) arXiv:hep-th/0602238; Phys. Lett. B 646, 258 (2007) arXiv:hep-ph/0605315].

[3] R. Bernabei et al. [DAMA Collaboration], Phys. Lett. B 480, 23 (2000); Eur. Phys. J. C 56, 333 (2008) arXiv:0804.2741 [astro-ph]].

[4] Z. Ahmed et al. [The CDMS-II Collaboration and CDMS-II Collaboration], arXiv:0912.3592 [astro-ph.CO].

[5] Ya. B. Zel'dovich and I. D. Novikov, Sov. Astron. 10, 602 (1966).

[6] S. W. Hawking, Commun. Math. Phys. 43, 199 (1975) [Erratum-ibid. 46, 206 (1976)].

[7] S. Hawking, Mon. Not. Roy. Astron. Soc. 152, 75 (1971).

[8] P. H. Frampton, JCAP 0910, 016 (2009) arXiv:0905.3632 [hep-th]].

[9] M. Crawford and D. N. Schramm, Nature 298, 538 (1982).

[10] S. W. Hawking, I. G. Moss and J. M. Stewart, Phys. Rev. D 26, 2681 (1982).

[11] D. La and P. J. Steinhardt, Phys. Lett. B 220, 375 (1989).

[12] S. W. Hawking, Phys. Lett. B 231, 237 (1989).

[13] A. Polnarev and R. Zembowicz, Phys. Rev. D 43, 1106 (1991).

[14] J. Garriga and M. Sakellariadou, Phys. Rev. D 48, 2502 (1993) arXiv:hep-th/9303024].

[15] R. R. Caldwell and P. Casper, Phys. Rev. D 53, 3002 (1996) arXiv:gr-qc/9509012.

[16] M. Kawasaki, N. Sugiyama and T. Yanagida, Phys. Rev. D 57, 6050 (1998) arXiv:hep-ph/9710259]; see also M. Kawasaki and T. Yanagida, Phys. Rev. D 59, 043512 (1999) arXiv:hep-ph/9807544.

[17] G. Lazarides and C. Panagiotakopoulos, Phys. Rev. D 52, 559 (1995).

[18] K. I. Izawa and T. Yanagida, Phys. Lett. B 393, 331 (1997).

[19] M. Yamaguchi and J. Yokoyama, Phys. Rev. D 70, 023513 (2004) arXiv:hep-ph/0402282.

[20] D. N. Spergel et al. [WMAP Collaboration], Astrophys. J. Suppl. 148, 175 (2003) arXiv:astro-ph/0302209. 
[21] M. Kawasaki, T. Takayama, M. Yamaguchi and J. Yokoyama, Phys. Rev. D 74, 043525 (2006) arXiv:hep-ph/0605271.

[22] T. Kawaguchi, M. Kawasaki, T. Takayama, M. Yamaguchi and J. Yokoyama, Mon. Not. Roy. Astron. Soc. 388, 1426 (2008) arXiv:0711.3886 [astro-ph]].

[23] C. Alcock et al. [MACHO Collaboration], Astrophys. J. 471, 774 (1996) arXiv:astro-ph/9604176]; C. Alcock et al. [MACHO Collaboration], Astrophys. J. 486, 697 (1997) arXiv:astro-ph/9606165].

[24] C. Renault et al. [EROS Collaboration], Astron. Astrophys. 324, L69 (1997) arXiv:astro-ph/9612102]; Astron. Astrophys. 329, 522 (1998).

[25] R. A. Allsman et al. [(Macho Collaboration) C Alcock], Astrophys. J. 550, L169 (2001) arXiv:astro-ph/0011506.

[26] P. Tisserand et al. [EROS-2 Collaboration], Astron. Astrophys. 469, 387 (2007) arXiv:astro-ph/0607207.

[27] J. Yoo, J. Chaname and A. Gould, Astrophys. J. 601, 311 (2004) arXiv:astro-ph/0307437.

[28] D. P. Quinn, M. I. Wilkinson, M. J. Irwin, J. Marshall, A. Koch and V. Belokurov, Mon. Not. Roy. Astron. Soc. 396, L11 (2009). arXiv:0903.1644 [astro-ph.GA]].

[29] M. Ricotti, J. P. Ostriker and K. J. Mack, Astrophys. J. 680, 829 (2008) arXiv:0709.0524 [astro-ph]].

[30] See however, M. Kawasaki, F. Takahashi and T. Takahashi, Phys. Lett. B 605, 223 (2005) arXiv:astro-ph/0407631.

[31] N. Tetradis, Phys. Rev. D 57, 5997 (1998) arXiv:astro-ph/9707214.

[32] T. Totani, arXiv:0908.3295 [astro-ph.CO]. 